\title{
BIOLOGIA DE Leptoglossus zonatus (DALLAS) (HEMIPTERA: COREIDAE) ALIMENTADOS COM MILHO E SORGO
}

\author{
Walter J.R. Matrangolo ${ }^{1}$ José M. Waquil ${ }^{1}$
}

\begin{abstract}
Biology of Leptoglossus zonatus (Dallas) (Heteroptera: Coreidae) Feeding on Corn and Sorghum

The biology of Leptoglossus zonatus (Dallas) was studied in a incubator at $28 \pm 2{ }^{\circ} \mathrm{C}, 78$ $\pm 5 \%$ of RH and 12 hours of photophase. The colony started from 10 couples of insects colected in corn and sorghum fields. Nymphs were fed on sorghum or corn grains and adults were fed only on sorghum. The mean pre-mate and pre-oviposition periods were 32.2 and 42.6 days. In average, each female laid 5.5 egg masses with 15.2 eggs distributed in a single line. The incubation period was 9.6 days in average and the nymphs showed five instars in a period of 28.7 days on corn, and 31.6 days on sorghum. It was observed 1.1 female to each male and the adult longevity was 71 days to females and 54.3 days to males. The survivors of immature stages totalized $46.2 \%$ when nymphs were fed on corn, and $44.9 \%$ when were fed on sorghum.
\end{abstract}

KEY WORDS: Insect, Zea mays, Sorghum bicolor, leaf-footed bug.

\section{RESUMO}

A biologia de Leptoglossus zonatus (Dallas) foi estudada em uma incubadora a $28 \pm 2^{\circ} \mathrm{C}$, U.R. de $78 \pm 5 \%$ e fotofase de 12 horas. A colônia iniciou-se a partir de 10 casais coletados em campos de milho e sorgo. As ninfas tiveram como alimento milho ou sorgo e os adultos foram alimentados somente com sorgo. A média do período de pré-cópula e pré-oviposição foi respectivamente 32,2 e 42,6 dias. Em média, cada fềmea colocou 5,5 massas de ovos com 15,2 ovos distribuídos numa única linha. O período de incubação foi 9,6 dias em média e as ninfas passaram por cinco ínstares, num período de 28,7 dias em milho e 31,6 dias em sorgo. Foi observada a proporção de 1,1 fêmea para cada macho e a longevidade dos adultos foi 71 dias para as fêmeas e 54,3 dias para os machos. A sobrevivência dos estádios imaturos foi $46,2 \%$ quando as ninfas foram alimentadas em milho e $44,9 \%$ em sorgo.

PALAVRAS-CHAVE: Insecta, Zea mays, Sorghum bicolor, coreídeo.

Recebido em 12/08/93. Aceito em 27/09/94.

${ }^{1}$ EMBRAPA/CNPMS, Km 65 da Rodovia 424, 35701-970, Sete Lagoas, MG. 


\section{INTRODUÇÃO}

O gênero Leptoglossus é citado em alguns trabalhos (Jadhav et al. 1980, Schaefer \& Mitchell 1983)como sendo praga de plantas cultivadas, das famílias Gramineae, Leguminosae, Rubiaceae, Rutaceae e Punicaceae. No Brasil, vários estudos foram feitos sobre o gênero (Amaral $\mathrm{F}^{\circ} \&$ Storti $\mathrm{F}^{\circ} 1976$, Amaral $\mathrm{F}^{\circ}$ \& Cajueiro 1977, Rolim et al. 1991).

No estado americano do Texas, Hall \& Teetes $(1982$ a,b) observaram constantes infestações de Leptoglossus phylopus (L.) em sorgo. Estes alimentam-se de hastes, brácteas e glumas de panículas de sorgo mas principalmente dos grãos. McMillian \& Wiseman (1972) também observaram infestações de L. phyllopus, em sorgo. Morril (1913) cita Leptoglossus zonatus (Dallas) alimentando-se de pomelos, laranja e pêssegos no Arizona (EUA). A espécie é importante praga de produtos agrícolas na Venezuela, segundo Osuna (1979). Em seu trabalho sobre a espécie, Panizzi (1989) cita a presença do inseto em várias culturas, entre elas, o milho e o sorgo. Este autor estudou o efeito de diferentes alimentos (milho, soja e feijão), na biologia de adultos e ninfas da espécie, tendo concluído que o milho é o alimento mais completo para L. zonatus. Conforme Sawazaki et al. (1989), L. zonatus além de causar falhas nas espigas de milho, contaminam os grãos com fungos como Fusarium moniliforme, Penicillium sp. e Cephalosporium sp. e observaram ainda a espécie causando dano em laranja, tomate e sorgo. Kubo \& Batista $\mathrm{F}^{\circ}(1992)$ afirmaram que os danos de L. zonatus em laranja, caracterizados por manchas circulares, atingiram $91 \%$ dos frutos.

A presença desse inseto em campos de milho e sorgo em Sete Lagoas, MG, e vários comunicados da ocorrência dessa espécie em diferentes estados da região Centro-sul do Brasil, motivaram esse trabalho. Foram então feitas observações de diversos parâmetros biológicos do inseto, utilizando-se como alimento milho e sorgo.

\section{MATERIAL E MÉTODOS}

As observações foram feitas entre os meses de abril e setembro de 1990, no Laboratório de Criação de Insetos (LACRI) do Centro Nacional de Pesquisa de Milho e Sorgo, Sete Lagoas, MG. Os insetos foram mantidos em câmara incubadora, com temperatura de $28 \pm 2^{\circ} \mathrm{C}$, UR de $78 \pm 5 \%$ e fotofase de 12 horas. Foram coletados 10 casais de L. zonatus em campos de milho e sorgo, em Sete Lagoas, MG. Estes foram mantidos em gaiolas de PVC de 7,5 cm de diâmetro por $7,5 \mathrm{~cm}$ de altura, tendo como base uma placa de Petri forrada com papel filtro úmido. A outra extremidade foi vedada com malha de filó, sobre a qual foi depositado oalimento, coberto com tecido de organza. Os tecidos foram presos por um elástico. Esse método permitia a troca de alimento sem o contato direto com os insetos e proporcionava fácil acesso destes ao alimento. Os 10 casais coletados no campo foram alimentados com espiguetas de sorgo, com grãos na fase leitosa. Os ovos obtidos a partir desses casais produziram os insetos que serviram de base para as observações subsequentes. Metade das ninfas daí originadas foi alimentada com grãos de sorgo e a outra metade com grãos de milho, ambos na fase leitosa. Os adultos provenientes da primeira geração foram separados em casais. Teve-se o cuidado de não permitir cruzamento entre irmãos. Os dados referentes à fase reprodutiva do inseto, são provenientes da primeira progênie dos 10 casais iniciais. A falta de grãos de milho na fase leitosa não permitiu a observação do desenvolvimento dos insetos adultos nesse alimento. Com isso, a longevidade de adultos foi observada tendo apenas o sorgo como fonte de alimento. Os parâmetros observados foram os seguintes: período de incubação, razão sexual, períodos de pré-cópula, pré-oviposição, número de ovos e número de posturas por fêmea, viabilidade total das ninfas nos diferentes alimentos, número de instares e duração dafase ninfal e longevidade dos adultos. 


\section{RESULTADOS E DISCUSSÃO}

As fềmeas de $L$. zonatus geralmente são maiores que os machos, confirmando observações de Panizzi (1989), onde o parâmetro avaliado foi peso do inseto. Os ovos foram postos enfileirados, aderidos ao substrato, do mesmo modo como citado por Fernandes \& Grazia (1992), em estudos com a espécie. Essas observações também coincidem com aquelas feitas por Amaral $\mathrm{F}^{\circ} \&$ Cajueiro (1977), quando estudaram a biologia de Veneza (=Leptoglossus) stigma (Herbst) e por Amaral $\mathrm{F}^{\circ} \&$ Storti $\mathrm{F}^{\circ}$ (1976) em estudos sobre L. gonagra (Fabricius). Jadhav et al. (1980) observaram o mesmo para L. membranadus (Fabricius) o que parece ser uma característica inerente ao gênero.

Inicialmente os ovos tem coloração esverdeada, tornando-se definitivamente marrons após algumas horas, como citado por Fernandes \& Grazia (1992). O período de incubação (de 710 ovos observados) teve uma média de 9,6 dias, período esse maior que o de L. gonagra obtido por Amaral $\mathrm{F}^{\circ} \&$ Storti $\mathrm{F}^{\circ}(1976)$ com média de 8,2 e menor que em $V$. stigma, onde Amaral $\mathrm{F}^{\circ}$ \& Cajueiro (1977) encontraram um período de incubação médio de 13,1 dias. As ninfas mantêm-se agrupadas até o final do $2^{\circ}$ estádio, após o que se dispersam pela gaiola reduzindo então a velocidade de movimento. A observação se assemelha àquela feita por Amaral $\mathrm{F}^{\circ}$ \& Cajueiro (1977), em L. stigma, assim como em trabalho de Wheeler Jr. \& Miller (1990), sobre L. fulvicornis (Westwood). Decorridos 28,7 dias em média, as ninfas de primeiro estádio atingiram a fase adulta, quando alimentadas com milho; tendo sorgo como alimento, esse período foi de 31,6 dias. Foram observados cinco estádios em ambos os alimentos.

De um total de 225 ovos, 104 indivíduos chegaram a fase adulta $(46,2 \%)$ quando criados em milho. Já em sorgo, 180 atingiram a fase adulta (44,9\%), originadas de 401 ovos. Foi observada a proporção de 1,1 fêmea para cada macho. Foi observado que $70,3 \%$ das fêmeas alimentadas com sorgo ovipositaram pelo menos uma vez; Panizzi (1989) obteve 83,3\%, quando as fêmeas da mesma espécie foram alimentadas com milho. Este autor observou ganho de peso muito superior quando a fonte de alimento foi grãos de milho, comparado com vagem de soja e vagem de feijão, ficando evidenciada a melhor qualidade nutricional do primeiro alimento para L. zonatus.

Tabela 1. Parâmetros biológicos de Leptoglossus zonatus, mantidos em laboratório e alimentados com milho e sorgo.

\begin{tabular}{lrrrr}
\hline Parâmetros & Média & Amplitude & $\begin{array}{c}\text { Int. confiança } \\
95 \%\end{array}$ & Número \\
\hline Incubação (dias) & 9,6 & $8-12$ & 0,07 & 710 \\
Pré-cópula (dias) & 32,2 & $14-49$ & 5,40 & 14 \\
Pré-oviposição (dias) & 42,6 & $19-58$ & 7,50 & 14 \\
Ovos/fêmea ( ${ }^{\circ}$ ) & 96,2 & $3-270$ & 42,50 & 18 \\
Postura/fêmea ( $\mathrm{n}^{\circ}$ ) & 5,5 & $1-121$ & 1,70 & 18 \\
Ovos/postura $\left(\mathrm{n}^{\circ}\right)$ & 15,2 & $3-33$ & 1,70 & 92 \\
Estágio ninfal(milho) & 28,7 & $22-31,5$ & 0,30 & 210 \\
Estágio ninfal(sorgo) & 31,6 & $27-35,5$ & 0,36 & 180 \\
Longevidade fêmea(dias) & 71,0 & $44-122$ & 12,00 & 23 \\
Longevidade macho(dias) & 54,3 & $23-76$ & 5,60 & 22 \\
\hline
\end{tabular}


O período de pré-cópula em $L$. zonatus foi em média de 32,2 dias. O mesmo parâmetro em L. gonagra foi 7,8 dias, segundo Amaral $\mathrm{F}^{\circ} \&$ Storti $\mathrm{F}^{\circ}$ (1976). O período médio de préoviposição encontrado foi de 42,6 dias, em 14 fêmeas de $L$. zonatus. Amaral $\mathrm{F}^{\circ} \&$ Storti $\mathrm{F}^{\circ}$ (1976) encontraram uma média de 20,1 dias, para o mesmo parâmetro em L. gonagra (Tabela 1).

A fêmea de $L$. zonatus ovipositou até 122 dias de idade. Observou-se a eclosão de ninfas provenientes de casais dos quais os machos haviam morrido 73 dias antes. Os parâmetros ovos/ fêmea, postura/fêmea e ovos/postura, obtidos a partir de 18 fêmeas nascidas no laboratório, apresentaram as seguintes médias, respectivamente: 96,2; 5,5 e 15,2, num total de 92 posturas observadas (Tabela 1). Os mesmos parâmetros encontrados por Amaral $\mathrm{F}^{\circ}$ \& Storti $\mathrm{F}^{\circ}$ (1976) em 11 fêmeas de L. gonagra apresentam os seguintes dados, respectivamente: 61,5; 6,2 e 13,1, em 139 posturas observadas. Panizzi (1989) verificou que, quando alimentadas com sementes de milho verde, cada fêmea de $L$. zonatus ovipositou em média 5,2 massas de ovos, com uma média de 107,6 ovos ao longo de sua vida.

Quando alimentados apenas com sorgo na fase de adultos, foram obtidas as seguintes longevidades para machos e fêmeas, respectivamente: 54,3 dias e 71,0 dias (Tabela 1). A longevidade para adultos de L. zonatus, em trabalho de Panizzi (1989) foi de 65 dias em média, para machos e fêmeas.

Ninfas e adultos de L. zonatus foram observados em plantas de romã, em pomares em Sete Lagoas. Adultos alimentando-se e em posição de cópula (postura especular genitáliagenitália), foram encontrados frequentemente no campo. O mesmo foi reportado por Amaral $\mathrm{F}^{\circ} \&$ Cajueiro (1977), para V. stigma. Na maioria das observações em milho, os adultos de $L$. zonatus foram encontrados sobre espigas, com o estilete atravessando a palha que protege os grãos, dos quais estavam se alimentando. Já as ninfas foram encontradas em sua maioria nas folhas de milho. Em sorgo, ninfas e adultos se postaram nas panículas.

\section{AGRADECIMENTOS}

Agradecemos a Dra. Maria C. Del Vecchio, Instituto Biológico de São Paulo, pelas sugestões na elaboração da versão final do manuscrito.

\section{LITERATURA CITADA}

Amaral $F^{\circ}$, B.F. do \& A. Storti $F^{\circ}$. 1976. Estudos biológicos sobre Leptoglossus gonagra (Fabricius, 1775), (Coreidae: Hemíptera)em laboratório. An. Soc. Entomol. Brasil 5: 130137.

Amaral Fo, B.F. do \& I.V.M. Cajueiro. 1977. Observação sobre o ciclo biológico de Veneza stigma (Herbst, 1784) Osuna, 1975 (Hemíptera: Coreidae) em laboratório. An. Soc. Entomol. Brasil 6: 164-172.

Fernandes, J.A.M. \& Grazia, J. 1992. Estudo das estágios imaturos de Leptoglossuszonatus (Dallas, 1852) (Heteroptera: Coreidae). An. Soc. Entomol. Brasil 21: 179-188.

Hall, D.G., IV \& G.L. Teetes. 1982a. Yield loss-density relationship of four species of panicle feeding bugs in sorghum. Environ. Entomol. 11: 738-741. 
Hall, D.G., IV \& G.L. Teetes. 1982b. Damage to grain sorghum by southern green stink bug, conchuela and leaf-footed bug. J. Econ. Entomol. 75: 620-625.

Jadhav, L.D., M.V. Kadan, D.S. Agri \& R.N. Pokharkar. 1980. Observation on the biology of leaf-footed plant bug Leptoglossus membranaceus Fabricius, a pest of pomegranate. Bull. Ent. 21: 79-82.

Kubo, R.K. \& Batista Fo, A. 1992. Ocorrência e danos provocados por Leptoglossus zonatus (Dallas, 1852) (Hemíptera: Coreidae) em citrus. An. Soc. Entomol. Brasil 21: 467-470.

McMillian, W.W. \& B.R. Wiseman. 1972. Insect species present on sorghum heads of various stages of maturity. Georgia Entomol. Soc. 7: 179-182.

Morril, A.W. 1913. Entomological pioneering in Arizona. J. Econ. Entomol. 6: 185-195.

Osuna, E. 1979. El torax y su musculature en Leptoglossus zonatus Dallas, (Heteroptera: Coreidae). Rev. Fac. Agron. Univ. Cent. Venez. 10: 455-505.

Panizzi, A.R. 1989. Desempenho de ninfas e adultos de Leptoglossus zonatus (Dallas, 1852) (Hemiptera: Coreidae) em diferentes alimentos. An. Soc. Entomol. Brasil 18: 375-389.

Rolim, A.E., R.L.A. Rolim, M.A.M. Leal \& D.T.M. Oliveira. 1991. Notas sobre o desenvolvimento de Leptoglossus stigmus (Herbst) (Hemiptera: Coreidae), uma praga do urucueiro, Bixa orellana L., no estado do Ceará. In Resumos Congresso Brasileiro de Entomologia, 13, Recife, 672p.

Sawazaki, E., C.J. Rossetto, G.M. Fantini \& A. Petinelli Junior. 1989. Leptoglossus zonatus (Dallas, 1852) nova praga do milho. In Resumos Reunião Anual do Instituto Biológico, 2, São Paulo, 91p.

Schaefer, C.W. \& P.L. Mitchell. 1983. Food plants of the Coreidea(Hemiptera: Heteroptera). Ann. Entomol. Soc. Am. 76: 591-615.

Wheeler Jr., A.G. \& G.L. Miller. 1990. Leptoglossus fulvicornis (Heteroptera: Coreidae), a specialist on magnolia fruits: Seasonal history, habits and descriptions of immature stages. Ann. Entomol. Soc. Am. 83: 753-765. 